\title{
Turning solid aluminium transparent by intense soft X-ray photoionization
}

\author{
Bob Nagler et al.
}

Saturable absorption is a phenomenon readily seen in the optical and infrared wavelengths. It has never been observed in core-electron transitions owing to the short lifetime of the excited states involved and the high intensities of the soft $X$-rays needed. We report saturable absorption of an L-shell transition in aluminium using record intensities over $10^{16} \mathrm{~W} \mathrm{~cm}^{-2}$ at a photon energy of $92 \mathrm{eV}$. From a consideration of the relevant timescales, we infer that immediately after the $X$-rays have passed, the sample is in an exotic state where all of the aluminium atoms have an L-shell hole, and the valence band has approximately a $9 \mathrm{eV}$ temperature, whereas the atoms are still on their crystallographic positions. Subsequently, Auger decay heats the material to the warm dense matter regime, at around $25 \mathrm{eV}$ temperatures. The method is an ideal candidate to study homogeneous warm dense matter, highly relevant to planetary science, astrophysics and inertial confinement fusion.

S aturable absorption, the decrease in the absorption of light with increasing intensity, is a well-known effect in the visible and near-visible region of the electromagnetic spectrum ${ }^{1}$, and is a widely exploited phenomenon in laser technology. Although there are many ways to induce this effect, in the simplest two-level system it will occur when the population of the lower, absorbing level is severely depleted, which requires light intensities sufficiently high to overcome relaxation from the upper level. Here, we report on the production of saturable absorption of a metal in the soft X-ray regime by the creation of highly uniform warm dense conditions, a regime that is of great interest in high-pressure science $^{2,3}$, the geophysics of large planets ${ }^{4,5}$, astrophysics ${ }^{6}$, plasma production and inertial confinement fusion ${ }^{7}$. Furthermore, the process by which the saturation of the absorption occurs will lead, after the X-ray pulse, to the storage of about $100 \mathrm{eV}$ per atom, which in turn evolves to a warm dense state. This manner of creation is unique as it requires intense, subpicosecond, soft X-rays. As such, it has not hitherto been observed in this region of the spectrum, owing both to the lack of high-intensity sources, and the rapid recombination times associated with such high photon energies. However, with the advent of new fourth-generation X-ray light sources, including the free-electron laser in $\mathrm{Hamburg}^{8}$ (FLASH), soft X-ray intensities that have previously remained the province of high-power optical lasers can now be produced. Experiments at such high intensities using gas jets have already exhibited novel absorption phenomena ${ }^{9}$, and the possibility of irradiating solid samples with intense soft and hard X-rays has aroused interest as a possible means of producing warm dense matter (WDM) at known atomic densities $^{10,11}$.

We present the first measurements of the absorption coefficient of solid samples subject to subpicosecond soft X-ray pulses with intensities up to and in excess of $10^{16} \mathrm{~W} \mathrm{~cm}^{-2}$, two orders of magnitude higher than could previously be obtained. The experiment has two phases: the first occurs during the $15 \mathrm{fs}$ freeelectron laser (FEL) pulse, whereas the second occurs after the pulse. During the first phase the sample absorbs predominantly through inner L-shell photoionization. As the pulse intensity is increased, the rate of photoionization surpasses the recombination rate so that the inner-shell absorbers are severely depleted, leading to a saturation of the absorption (that is, the aluminium becomes highly

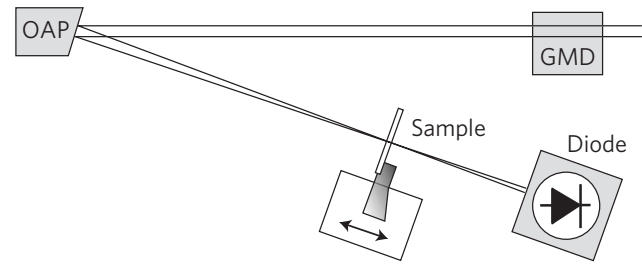

Figure 1 | Schematic diagram of the experimental set-up. The energy of the incoming beam is measured by the gas-monitor detector (GMD). The beam is focused with an off-axis multilayer-coated Mo/Si off-axis parabola (OAP) onto the sample. The transmitted energy is measured by a silicon photodiode.

transmissive). In the second phase, the photoionized electrons decay by two paths, radiative and Auger (radiationless) decay. In principle, the former provides spectral information on the temperature of the valence band, whereas the latter transfers energy to the valence electrons that is effectively stored for times of the order of several picoseconds (the electron-phonon coupling time). We observe the first phase by measuring the transmission of the pulse over a wide range of fluences. In separate experiments, covering only the lowest fluences used, we have observed the second phase by spectroscopy.

The experiment, shown in schematic form in Fig. 1, was carried out using FLASH operating at a wavelength of $13.5 \mathrm{~nm}$, that is, a photon energy of $92 \mathrm{eV}$. The laser produced pulses of radiation containing between 10 and $50 \mu \mathrm{J}$ per pulse in a pulse length of the order of $15 \mathrm{fs}$ (ref. 8) at a repetition rate of $5 \mathrm{~Hz}$. The highly collimated beam of $5 \mathrm{~mm}$ diameter was focused onto solid samples using a $\mathrm{Mo} / \mathrm{Si}$ multilayer-coated off-axis parabola with a focal length of $269 \mathrm{~mm}$, and reflectivity of $48 \%$. Focal spot sizes were determined to be $1.5 \mu \mathrm{m}$ at best focus by using Nomarski optical microscopy and atomic force microscopy to look at damage craters induced by the radiation on poly(methyl methacrylate) (ref. 12). The aluminium samples were placed at nine different positions from best focus, with the full-width at half-maximum diameter of the laser spot varying from $1.5 \mu \mathrm{m}$ at best focus, up to $45 \mu \mathrm{m}$. The energy within each pulse was measured with a gas-monitor detector $^{13}$ (GMD). By variation of both the sample position and laser energies, three orders of magnitude of laser fluence

\footnotetext{
${ }^{*}$ A full list of authors and their affiliations appears at the end of the paper.
} 
were scanned. The transmission of 53-nm-thick aluminium was recorded as a function of laser fluence using a silicon photodiode, which was calibrated with the GMD energies in the absence of a sample. The calibration shows a linear correlation of the GMD signal with respect to the photodiodes. However, this measurement shows a $\pm 10 \%$ shot-to-shot error, which limits the accuracy of the measured absorption to $\pm 10 \%$ on a shot-to-shot basis. Statistically larger variations between GMD and photodiode readings were found for the lowest beam energies $(\leq 10 \mu \mathrm{J})$, and such unusually low-energy shots are excluded from the analysis.

With an unexposed region of the aluminium sample in place, the transmission is measured by comparing the energies recorded by the calibrated photodiode and GMD for every shot. Transmission as a function of fluence is shown in Fig. 2. Saturable absorption can readily be seen in the experimental data, with an increase in transmission from $15 \%$ at the lowest fluence of $0.3 \mathrm{~J} \mathrm{~cm}^{-2}$, to $65 \%$ at a fluence of $2 \times 10^{2} \mathrm{~J} \mathrm{~cm}^{-2}$. Given a pulse length of $15 \mathrm{fs}$, these limits correspond to irradiances of $2 \times 10^{13} \mathrm{~W} \mathrm{~cm}^{-2}$ and $1.5 \times 10^{16} \mathrm{~W} \mathrm{~cm}^{-2}$ respectively.

A theoretical model that explains this increase in transmission with fluence can be constructed by considering the absorption mechanisms and relaxation timescales pertinent to these extreme conditions. At standard temperature and pressure, solid aluminium has a filled-shell ionic core $\left(1 s^{2} 2 s^{2} 2 p^{6}\right)$ with the three free electrons (coming from the $3 s^{2}$ and $3 p$ atomic electrons) in the valence band. As such, it has a relatively deep L-edge. The $\mathrm{L}_{\text {III }}$ and $\mathrm{L}_{\mathrm{II}}$ edges lie below the photon energy, at 72.7 and $73.1 \mathrm{eV}$ respectively, whereas the $\mathrm{L}_{\mathrm{I}}$ lies at $117.8 \mathrm{eV}$ (ref. 14). Experimental values for the absorption coefficient of cold aluminium are well known in this region ${ }^{14}$. The contribution due to free-free absorption (that is, due to the valence electrons) in this region is very small compared with the photoionization, and we determine values for the photoionization absorption coefficient and the free-free coefficient as $27 \mu \mathrm{m}^{-1}$ and $0.2 \mu \mathrm{m}^{-1}$ respectively for cold $\mathrm{Al}$ (ref. 15). Therefore, for photons of energy $92 \mathrm{eV}$, almost all of the absorption is due to photoionization of L-shell electrons. At high intensities, the fraction of aluminium atoms with photoionized L-shell electrons is high, leading to depletion of L-shell absorption: after an electron is ejected from the L-shell of an atom, the L-edge of that particular atom will increase owing to reduced screening. The energy needed to eject a second L-shell electron is calculated to be $93 \mathrm{eV}$ (ref. 16), which is confirmed by measurements of Auger energies $^{16,17}$. Therefore, the FEL (at $92.5 \mathrm{eV}$ ) will not be able to create a second L-shell hole; the photoionization quenches and the absorption coefficient is heavily reduced to the value of the free-free absorption. Once an electron is ejected from the L-shell, the hole will be refilled by means of either radiative decay or the dominant Auger decay. However, the total lifetime of such an L-shell vacancy (which is much shorter than the radiative lifetime) is estimated to be around 40 fs (ref. 18), that is, long compared with the FLASH pulse length, but still short compared with any electron-phonon coupling time, or hydrodynamic motion. Thus, the loss of L-shell electrons during the initial part of the FLASH pulse results in reduced absorption during the rest of the pulse-an effect that is negligible at previously accessible soft X-ray intensities.

We can calculate the absorption of the FEL pulse versus its intensity by keeping track of the densities of the unexcited and photo excited aluminium cores as the pulse propagates through the foil, and scaling the bound-free and free-free contributions to the absorption accordingly. Thermal ionization of the L-shell does not occur, as it requires a temperature above $10 \mathrm{eV}$ at solid densities ${ }^{19}$. The resultant transmission as a function of fluence is plotted alongside the experimental data in Fig. 2. As the surface of aluminium foils readily oxidizes, we assume a $10 \mathrm{~nm}$ layer of aluminium oxide formed on either side of the foil ${ }^{20}$, which matches both our data at the lowest fluence and transmission

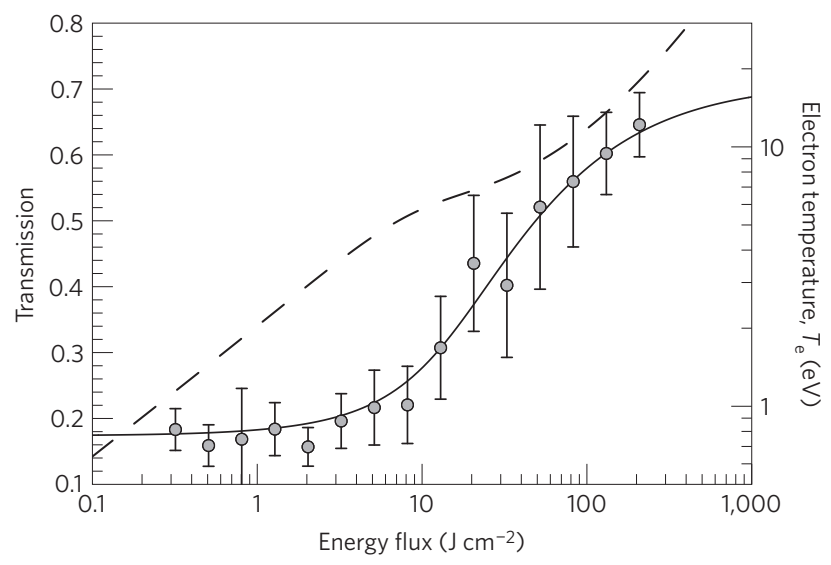

Figure 2 | Transmission of aluminium target as a function of fluence. The circles are the experimental data points with a $1 \sigma$ error bar; the solid line is the theoretical prediction (see text). The dashed curve is the predicted electron temperature in electronvolts (right $y$ axis) in the valence band after the FEL pulse has passed, but before the L-shell holes are filled and the Auger recombination heats the band further.

measurements carried out on the same sample at a synchrotron facility (Advanced Light Source) with the tabulated cold absorption of aluminium $^{14}$. The theoretical model shows excellent agreement with the experiment.

The photo-excited electrons have an energy of the order of $20 \mathrm{eV}$ above the original Fermi energy. Measured inelastic scattering lengths for such electrons in aluminium are between 5 and $10 \AA$ (ref. 21) corresponding to timescales of the order of $0.85-1.7 \mathrm{fs}$, in good agreement with calculations based on electron gas dielectric theory ${ }^{22}$. Thus, the photo-excited electrons rapidly lose their energy and thermalize with the initial cold valence electrons on a timescale considerably shorter than the FLASH pulse. As this electron thermalization time is short compared with the pulse length and Auger decay time, both the electron temperature and electron density immediately after the passage of the FLASH pulse can be calculated. The electron temperature is calculated by assuming that the excited electrons, which have a kinetic energy equal to the photon energy minus the energy of the bottom of the valence band, thermalize with the other valence electrons. This predicted electron temperature after the FLASH pulse has passed is plotted as a function of fluence in Fig. 2. We note that even at the highest fluences, where we expect to have four electrons per atom in the band, we estimate that the electron temperature at the end of the passage of the pulse is only of the order of $9 \mathrm{eV}$.

Subsequently, L-shell holes will be filled with valence band electrons. The dominant mechanism here will be Auger recombination (the radiative yield is only $0.2 \%$; ref. 18 ), which will heat the valence band electrons, as each Auger electron will receive about $70 \mathrm{eV}$ of energy. Therefore, over the lifetime of the excited L-shell state, we calculate that the electron temperature will increase from about $1 \mathrm{eV}$ to approximately $2 \mathrm{eV}$ for the lower fluences in Fig. 2, and from 9 to $25 \mathrm{eV}$ for the highest fluences.

Information about the electron distribution (and hence temperature) in the valence band can be inferred from soft X-ray emission spectra. We have measured such spectra in separate experiments corresponding to the lower end of the fluence regime shown in Fig. 2. In these experiments, carried out with a different set-up and a $29 \mu \mathrm{m}$ focal $\operatorname{spot}^{23}$, the integrated spectra of 1,800 shots were measured (see Fig. 3). The radiative recombination of electrons from the valence band to the $\mathrm{L}_{\mathrm{III}}$ or $\mathrm{L}_{\mathrm{II}}$ levels results in emission that ranges in energy from approximately $62 \mathrm{eV}$ (the energy difference between the L levels and the bottom of the valence band) to a thermally broadened region at an energy corresponding to the 


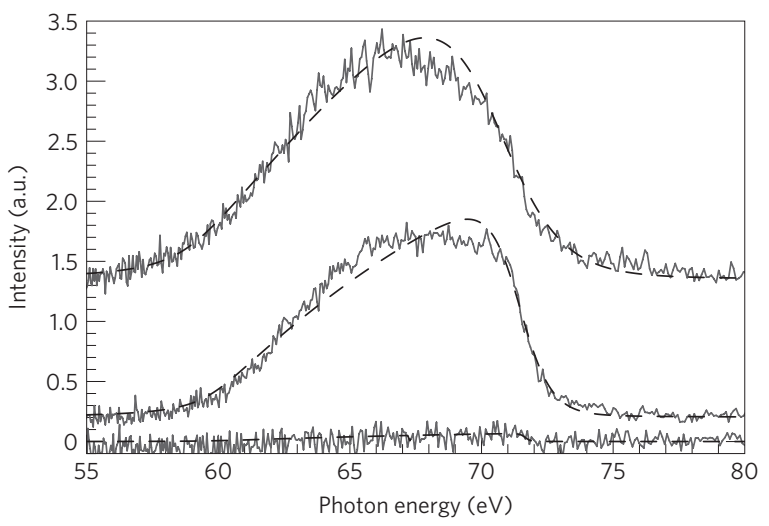

Figure 3 | Soft X-ray emission spectrum of solid aluminium. Experimental data (solid lines) for fluences from top to bottom of $0.6 \mathrm{~J} \mathrm{~cm}^{-2}, 0.3 \mathrm{~J} \mathrm{~cm}^{-2}$ and $0.04 \mathrm{~J} \mathrm{~cm}^{-2}$. Spectra are fitted (dashed lines) using equation (1) where we also include electron collision and Auger level broadening ${ }^{25}$, resulting in temperatures of respectively $1.2 \mathrm{eV}, 0.5 \mathrm{eV}$ and $0.1 \mathrm{eV}$. Spectra are offset for clarity.

difference between the Fermi energy and the L-shell—an energy of around the L-edge energy at $73 \mathrm{eV}$.

The line shape of the emission can be written as $^{24}$ :

$$
I(E) \sim \omega^{3} P(E) g(E) f_{T_{\mathrm{e}}}(E)
$$

where $\omega$ is the emission frequency, $P(E)$ is the square of the relevant transition matrix element, $g(E)$ is the free-electron density of states and $f_{T_{\mathrm{e}}}(E)$ is the Fermi function for electron temperature $T_{\mathrm{e}}$. In Fig. 3, experimentally recorded spectra are compared with theory based on equation (1), where we also take Auger broadening and broadening due to electron collisions into account ${ }^{25}$.

The soft X-ray emission is here seen to arise from the aluminium valence band from which we infer that the sample is still at solid density and the electrons at a temperature that can be estimated by the slope of the spectra on the high-energy edge. In this way, the electron temperature is inferred to be $1.2 \mathrm{eV}, 0.5 \mathrm{eV}$ and $0.1 \mathrm{eV}$ for fluences of respectively $0.6 \mathrm{~J} \mathrm{~cm}^{-2}, 0.3 \mathrm{~J} \mathrm{~cm}^{-2}$ and $0.04 \mathrm{~J} \mathrm{~cm}^{-2}-$ temperatures that are broadly in agreement with those calculated for the lower end of the fluence regime shown in Fig. 2. In fact they are slightly lower than the calculated temperatures, which may be due to the fact that the temperature is not uniform as a function of depth within the sample in this unsaturated regime. Evidently the collection of spectra at higher irradiances in future experiments affords the potential to learn directly about the electron temperature in this regime.

Returning to consider the response of the target at the highest fluences, we note that immediately after the passage of the FLASH pulse, where the high transmission indicates that almost every atom has an L-shell hole, we expect the aluminium ions to be close to their original lattice positions: the expected electronphonon coupling time is over a picosecond ${ }^{26}$ and even after receiving energy from the electrons, an aluminium ion with a temperature of a few electronvolts takes more than 50 fs to traverse a lattice spacing. Thus, at the instant the pulse has passed, we can infer that the aluminium is in an exotic, highly ionized, yet crystalline state, of which the physical properties (for example, band structure, equation of state, phonon spectrum and so on) are largely unknown. How this system will subsequently evolve into equilibrated WDM is still largely an open question, as this depends on electron-phonon coupling times and ion-ion interactions that have yet to be measured for these conditions. Measuring these crucial parameters is beyond the scope of the current work, and clearly one of the key experiments that must be carried out in the future.

The results presented here are of significant interest in the context of the creation of WDM by the irradiation of solid samples by high-intensity soft and hard X-ray radiation. Saturating an absorption edge enables very uniform heating of a sample: whereas the front part of the pulse is absorbed and heats the front of the sample, the back of the pulse will pass through this bleached region, heating the back of the sample to nearly the same temperature. For example, at $100 \mathrm{~J} \mathrm{~cm}^{-2}, 50 \%$ of the FEL pulse will be absorbed (see Fig. 2), but the temperature difference between the front and the back of the sample is calculated to be only $5 \%$. This compares favourably with experiment where thin samples are heated with high-intensity optical lasers. Although such experiments have yielded excellent results and increased our understanding of WDM significantly ${ }^{27,28}$, the sample thickness that can be uniformly heated in this way is ultimately limited by the electron ballistic depth of the hot electrons that the laser generates. For example in ref. 28, this ballistic depth is around $110 \mathrm{~nm}$, which would mean that the back of $52 \mathrm{~nm}$ aluminium targets is exposed to only $60 \%$ of the energy compared with the front of the target, which will never allow for uniform heating within $5 \%$ as in our current experiments. By saturating the absorption edge, we have efficiently created uniformly heated warm dense aluminium.

We have presented the first demonstration of saturable absorption induced by record soft X-ray intensities. These results are consistent with the ejection of an L-electron from almost all atoms in the sample on femtosecond timescales. Although each atom receives around $100 \mathrm{eV}$ from the photons, a consideration of the relative timescales of electron-electron inelastic scattering and Auger decay implies that the electrons in the valence band remain relatively cool before filling the L-shell holes by means of Auger decay. Soft X-ray emission data obtained at the lower end of the fluence regime show that in the future more detailed measurements of the electron temperature may be possible. By bleaching the L-edge transition in this way within $15 \mathrm{fs}$ (on a much shorter timescale than the picoseconds calculated to be necessary for electron-ion equilibration), we infer that we have created a very transient exotic state of highly ionized crystalline matter.

\section{Received 9 February 2009; accepted 17 June 2009;} published online 26 July 2009

\section{References}

1. Lewis, G. N., Lipkin, D. \& Magel, T. T. Reversible photochemical processes in rigid media. A study of the phosphorescent state. J. Am. Chem. Soc. 63, 3005-3018 (1941).

2. Davidson, R. Frontiers in High Energy Density Physics: The X-Games of Contemporary Science. (National Academies Press, 2003).

3. Frontiers for Discovery in High Energy Density Physics. Prepared for National Science and Technology Council, Committee on Science by the Interagency Working Group on the Physics of the Universe. (2004).

4. Guillot, T. Interiors of giant planets inside and outside the solar system. Science 286, 72-77 (1999).

5. Nettelmann, N. et al. Ab initio equation of state data for hydrogen, helium, and water and the internal structure of Jupiter. Astrophys. J. 683, 1217-1228 (2008).

6. Turner, M. Connecting Quarks with the Cosmos: Eleven Science Questions for the New Century (National Academies Press, 2003).

7. Lindl, J. D. et al. The physics basis for ignition using indirect-drive targets on the National Ignition Facility. Phys. Plasmas 11, 339-491 (2004).

8. Ackermann, W. et al. Operation of a free-electron laser from the extreme ultraviolet to the water window. Nature Photon. 1, 336-342 (2007).

9. Sorokin, A. A. et al. Photoelectric effect at ultrahigh intensities. Phys. Rev. Lett. 99, 213002 (2007).

10. Dyer, G., Bernstein, A., Cho, B. \& Osterholz, J. Equation-of-state measurement of dense plasmas heated with fast protons. Phys. Rev. Lett. 101, 015002 (2008).

11. Warm Dense Matter. <http://www.hiper-laser.org/science/ extremematerialsscience/warmdensematter.asp $>$.

12. Chalupský, J. et al. Characteristics of focused soft X-ray free-electron laser beam determined by ablation of organic molecular solids. Opt. Express 15, 6036-6043 (2007) 
13. Tiedtke, K. et al. Gas detectors for X-ray lasers. J. Appl. Phys. 103, 094511 (2008).

14. Henke, B. L., Gullikson, E. M. \& Davis, J. C. X-Ray Interactions: Photoabsorption, scattering, transmission, and reflection at $E=50-30,000 \mathrm{eV}$, $Z=1-92$. Atomic Data Nucl. Data Tables 54, 181-342 (1993).

15. Vinko, S. M. et al. Free-free opacity in warm dense aluminum. High Energy Density Phys. doi: 10.1016/j.hedp.2009.04.004 (2009, in the press).

16. Dufour, G., Mariot, J., Nilsson-Jatko, P. \& Karnatak, R. K-LL auger spectrum of aluminium. Phys. Scr. 13, 370-372 (1976).

17. Hannah, P. H. \& Weightman, P. The spectrum of $\mathrm{Al} \mathrm{KL}_{2,3}-\mathrm{L}_{2,3}^{2} \mathrm{~V}$ satellite Auger transitions. J. Phys. C 18, L239-L243 (1985).

18. Almbladh, C., Morales, A. \& Grossmann, G. Theory of Auger core-valence-valence processes in simple metals. I. Total yields and core-level widths. Phys. Rev. B 39, 3489-3502 (1989).

19. Kim, D. \& Kim, I. Calculation of ionization balance and electrical conductivity in nonideal aluminum plasma. Phys. Rev. E 68, 056410 (2003)

20. Wefers, K. Properties and characterization of surface oxides on aluminum alloys. Aluminum 57, 722-726 (1981).

21. Seah, M. P. \& Dench, W. A. Quantitative electron spectroscopy of surfaces: A standard database for electron inelastic mean free paths in solids. Surf. Interface Anal. 1, 2-11 (1979).

22. Ashley, J., Tung, C. \& Ritchie, R. Electron inelastic mean free paths and energy losses in solids: I. Aluminum metal. Surf. Sci. 81, 409-426 (1979).

23. Zastrau, U. et al. Bremsstrahlung and line spectroscopy of warm dense aluminum plasma heated by xuv free-electron-laser. Phys. Rev. E $\mathbf{7 8}$ 066406 (2008).

24. Fabian, D., Watson, L. \& Marshall, C. Soft X-ray spectroscopy and the electronic structure of solids. Rep. Prog. Phys. 34, 601-696 (1972).

25. Pirenne, J. \& Longe, P. Contribution of the double electron transitions to the soft X-ray emission bands of metals. Physica 30, 277-292 (1964).

26. Siwick, B., Dwyer, J., Jordan, R. \& Miller, R. An atomic-level view of melting using femtosecond electron diffraction. Science 302, 1382-1385 (2003)
27. Ernstorfer, R., Harb, M., Hebeisen, C. \& Sciaini, G. The formation of warm dense matter: Experimental evidence for electronic bond hardening in gold. Science 323, 1033-1037 (2009).

28. Ping, Y., Hanson, D., Koslow, I. \& Ogitsu, T. Broadband dielectric function of nonequilibrium warm dense gold. Phys. Rev. Lett. 96, 255003 (2006).

\section{Acknowledgements}

The authors want to acknowledge K. Budil of LLNL for assistance in support in funding, and acknowledge support for access to FLASH by DESY and the European Community under contract RII3-CT-2004-506008 (IA-SFS). The authors from Universität Rostock are supported by the Deutsche Forschungsgemeinschaft within SFB 652, B.N. by the EU Marie-Curie RTN 'FLASH', S.M.V. by EPSRC/STFC, W.M. by AWE, K.S. by the Slovak Grant Agency for Science (Grant No. 2/7196/27) and L.J., J.C., J.Ch. and V.H. by the Czech Ministry of Education (grants LC510, LC528 and LA08024) and Academy of Sciences of the Czech Republic (Z10100523, IAA400100701, and KAN 300100702). Technical assistance by A. Aquila, J. Meyer-Illse and E. M. Gullikson (LBNL) during the ALS beamtime is greatly appreciated. Operation of the Advanced Light Source was supported by the Director, Office of Science, Office of Basic Energy Sciences, of the US Department of Energy under Contract No. DE-AC03-76SF00098. We gratefully acknowledge financial support by the German Federal Ministry for Education and Research through project FSP 301-FLASH, and from the Ministry of Science and Higher Education of Poland through grant SPB No. DESY/68/2007. This work was in part carried out under the auspices of the US Department of Energy by Lawrence Livermore National Laboratory under Contract DE-AC52-07NA27344, and in part supported by grants 08-ERI-002 and 08-LW-004.

\section{Author contributions}

All authors contributed extensively to the work presented in this article.

\section{Additional information}

Reprints and permissions information is available online at http://npg.nature.com/ reprintsandpermissions. Correspondence and requests for materials should be addressed to B.N.

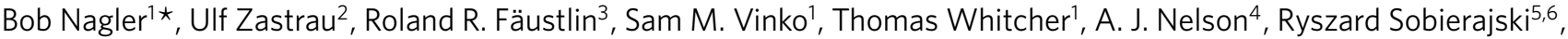
Jacek Krzywinski ${ }^{7}$, Jaromir Chalupsky ${ }^{8}$, Elsa Abreu9 ${ }^{9}$, Saša Bajt ${ }^{3}$, Thomas Bornath ${ }^{10}$, Tomas Burian ${ }^{8}$, Henry Chapman ${ }^{11,12}$, Jaroslav Cihelka ${ }^{8}$, Tilo Döppner ${ }^{4}$, Stefan Düsterer ${ }^{3}$, Thomas Dzelzainis ${ }^{13}$, Marta Fajardo ${ }^{9}$, Eckhart Förster², Carsten Fortmann ${ }^{10}$, Eric Galtier ${ }^{14}$, Siegfried H. Glenzer ${ }^{4}$, Sebastian Göde ${ }^{10}$, Gianluca Gregori ${ }^{1}$, Vera Hajkova ${ }^{8}$, Phil Heimann ${ }^{15}$, Libor Juha ${ }^{8}$, Marek Jurek ${ }^{5}$, Fida Y. Khattak ${ }^{16}$, Ali Reza Khorsand ${ }^{6}$, Dorota Klinger ${ }^{5}$, Michaela Kozlova ${ }^{9}$, Tim Laarmann ${ }^{3}$, Hae Ja Lee ${ }^{17}$, Richard W. Lee ${ }^{4}$, Karl-Heinz Meiwes-Broer ${ }^{10}$, Pascal Mercere ${ }^{18}$, William J. Murphy ${ }^{1}$, Andreas Przystawik ${ }^{10}$, Ronald Redmer ${ }^{10}$, Heidi Reinholz ${ }^{10}$, David Riley ${ }^{11}$, Gerd Röpke ${ }^{10}$, Frank Rosmej ${ }^{12}$, Karel Saks ${ }^{19}$, Romain Schott ${ }^{12}$, Robert Thiele ${ }^{10}$, Josef Tiggesbäumker ${ }^{10}$, Sven Toleikis ${ }^{3}$, Thomas Tschentscher ${ }^{20}$, Ingo Uschmann², Hubert J. Vollmer ${ }^{4}$ and Justin S. Wark

${ }^{1}$ Department of Physics, Clarendon Laboratory, University of Oxford, Parks Road, Oxford, OX1 3PU, UK, ${ }^{2}$ Institut für Optik und Quantenelektronik, Friedrich-Schiller-Universität, Max-Wien-Platz 1, 07743 Jena, Germany, ${ }^{3}$ Deutsches Elektronen-Synchrotron DESY, Notkestrasse 85, 22607 Hamburg, Germany, ${ }^{4}$ Lawrence Livermore National Laboratory, 7000 East Avenue, Livermore, California 94550, USA, ${ }^{5}$ Institute of Physics, Polish Academy of Sciences, Al. Lotnikow 32/46, 02-668 Warsaw, Poland, ${ }^{6}$ FOM-Institute for Plasma Physics Rijnhuizen, NL-3430 BE Nieuwegein, The Netherlands, ${ }^{7}$ SLAC National Accelerator Laboratory, 2575 Sand Hill Road, Menlo Park, California 94025, USA, ${ }^{8}$ Institute of Physics, Academy of Sciences of the Czech Republic, Na Slovance 2, 18221 Prague 8, Czech Republic, ${ }^{9}$ GoLP/IPFN, Instituto Superior Tecnico, 1049-001 Lisboa, Portugal, ${ }^{10}$ Institut für Physik, Universität Rostock, 18051 Rostock, Germany, ${ }^{11}$ Centre for Free-Electron Laser Science, DESY, 85 Notkestrasse, 22607 Hamburg, Germany, ${ }^{12}$ Universität Hamburg, Luruper Chaussee 149, 22761 Hamburg, Germany, ${ }^{13}$ Queen's University Belfast, University Road, Belfast, BT7 1NN, Northern Ireland, UK, ${ }^{14}$ UPMC, 4 place Jussieu 75005 Paris, France, ${ }^{15}$ Lawrence Berkeley National Laboratory, 1 Cyclotron Road, California 94720, USA, ${ }^{16}$ Department of Physics, Kohat University of Science and Technology, Kohat-26000, NWFP, Pakistan, ${ }^{17}$ Department of Physics, 366 LeConte Hall, University of California, Berkeley, California 94720, USA, ${ }^{18}$ SOLEIL, L'Orme des Merisiers Saint-Aubin, BP 4891192 GIF-sur-YVETTE, France, ${ }^{19}$ Institute of Materials Research, Slovak Academy of Sciences, Watsonova 47, 04001 Kosice, Slovak Republic, ${ }^{20}$ European XFEL Project team, DESY, Notkestrasse 85, 22607 Hamburg, Germany. *e-mail: Bob.Nagler@physics.ox.ac.uk. 